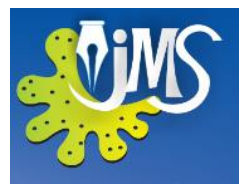

\title{
User-Age Classification Using Touch Gestures on Smartphones
}

\author{
${ }^{1}$ Suleyman AL-Showarah, ${ }^{1}$ Naseer AL-Jawad and ${ }^{1}$ Harin Sellahewa \\ ${ }^{1}$ Applied Computing Department, University of Buckingham, UK
}

\begin{abstract}
In this paper we investigated the possibility of classifying users' age-group using gesture-based features on smartphones. The features used were gesture accuracy, speed, movement time, and finger/force pressure. Nearest Neighbour classification was used to classify a given user's age-group. The 50 participants involved in this research included 25 elderly and 25 younger users. User-dependent and user-independent age-group classification scenarios were considered. On each scenario, two types of analysis were considered; using a single-feature and combined-features to represent a user-age group. The results revealed that classification accuracy was relatively higher for the younger age group than the elderly age group. Also, a higher classification accuracy was achieved on the small smartphone than on mini-tablets. The results also showed that the classification accuracy increases when combining the gesture features in to a single representation as opposed to using a single gesture feature.
\end{abstract}

KEYWORDS: User's age-group classification, security, finger on touchscreen

Corresponding author: Harin Sellahewa, eMail: harin.sellahewa@buckingham.ac.uk 


\section{INTRODUCTION}

A smartphone's interface plays an important role in the way in which we interact with its applications effectively and efficiently. Such interactions can be difficult when interfaces are less intuitive use, or when the users have less ability, experience and knowledge about how to use the technology (Pattison \& Stedmon, 2006). Hence, new technologies such as smartphones can be more difficult to use by people who cannot cope with technology's rapid evolution, or by people with physiological or cognitive deficits such as the elderly (Arnott et al., 2004).

Unfortunately, technology interfaces are not universally usable and, although there is a growing population of people who are motivated to use the technology, they find it physically difficult to do so (Hurst et al., 2008 \& Stößel, 2012). One of the main reasons designs are inaccessible is that they treat all users the same, and usually do not know much about each individual user's ability (Arnott et al., 2004).

In order to increase the usability of smartphones and tablets for elderly people, we conduct a research study on smartphones to investigate the possibility of classifying user age. The aim of this research is to provide evidence for the possibility of classifying users' age-group based on gesture-based features on smartphones. This particular study is part of a wider research study on the effects of ageing on smartphone and tablet usability (Al-Showarah, 2015).

The outcomes of this research could be used as a system to adapt itself to let users interact with technology based on their agerelated abilities, i.e., the system will turn into a particular setting based on its current user's agegroup. This could be particularly helpful to users who are unable to setup their own smartphone, tablet or a similar device to their personal preferences or for public systems that could be used by different users at different times.
This research propose the use of gesture accuracy (Acc), speed, movement time (MT), and finger/force pressure (FP) as touch gesturebased features to represent user age groups. A simple Nearest Neighbour (NN) classification will be used to classify a user as belonging to younger or elderly age group.

The rest of the paper is organised as follows: a literature review is presented in Section II; the methodology is discussed in Section III; Section IV explains the experiment design and procedure; Section V introduces the gesture features; Section VI introduces the user age-group classification process; Section VII presents experiment results and discussions which is followed by Section VII where we offer our concluding remarks and highlight future direction of work.

\subsection{Literature Review}

With the widespread use of touch-screen devices, analyzing finger movement behaviour on touch screens has become an interesting research topic. For example, finger movement behaviour could be used to authenticate or to control PIN-based interfaces used in most smartphones (Stößel, 2012 \& Sultana et al., 2013).

In most similar work, Hurst et al., (2008) conducted a study aimed at distinguishing between sub movements pointing for data collected from 8 younger adults (20-30 years old), 8 adults (35-65 years old), 7 older adults (70+ years old), and 6 participants with Parkinson's Disease (48-63 years old). This study is considered as the second stage of the study (Keates et al., 2005) that was conducted to examine the effects of age and Parkinson's disease on cursor positioning using a mouse. The specific features they used in the dataset are: 1) number of times if the task was performed correctly, and 2) time movement needed to complete the task. 
The statistical analysis showed that Hurst et al. were able to distinguish pointing behaviours among users. Based on Decision Tree, the statistical result between adults group vs. older adults group was at $93.8 \%$, adults group vs. younger adults group was at $59.3 .8 \%$, and younger adults group vs. older adults group was at $93.3 \%$ classification accuracy.

Sultana and Moffatt (2013) conducted a study to evaluate four algorithms; Decision Trees, Neural Networks, Naïve Bayesian Networks, and Rule Induction for identifying errors from sub-movement behaviour using penbased data for older adults (12 users, age range 65-86 years old), and younger (12 users, age range 19-29 years old). This study is considered as the second stage of the study (Moffatt, 2010) that conducted to reduce pen-based errors for elderly users from sub-movement behaviour. The results of the study in (Moffatt, 2010) were analysed based on observation. In order to distinguish errors from sub-movement of older users, there were three training datasets from the collected data, as follows: 1) older users, 2) younger users, 3) all users, and they labeled each data in the all databases as "Error" and "NoError". In their study, four algorithms were used; Decision Trees, Neural Networks, Naïve Bayesian Networks, and Rule Induction (RI) algorithms for all training datasets.

The results showed that each algorithm yielded a classification accuracy rate of around $90 \%$, while the Naïve Bayesian Networks provided the best classification accuracy between all algorithms. Classification accuracy for truly predicted errors for elderly was high compared with other age groups on all algorithms used.

There are number of other studies conducted on small and large touch screen sizes for elderly, but all these we came across (e.g. Stößel et al., 2009 \& and Stößel et al., 2012) were conducted to investigate the effect of ageing on touch screen.

\section{METHODOLOGY}

\section{a) Experiments Structure}

Error! Reference source not found.Figure 1 gives an overall view of the experiment setup for users of two age groups; $\mathrm{EG}$, and YG. Each participant was involved only on one smartphone screen size to avoid any influence of familiarity on the participant's performance.

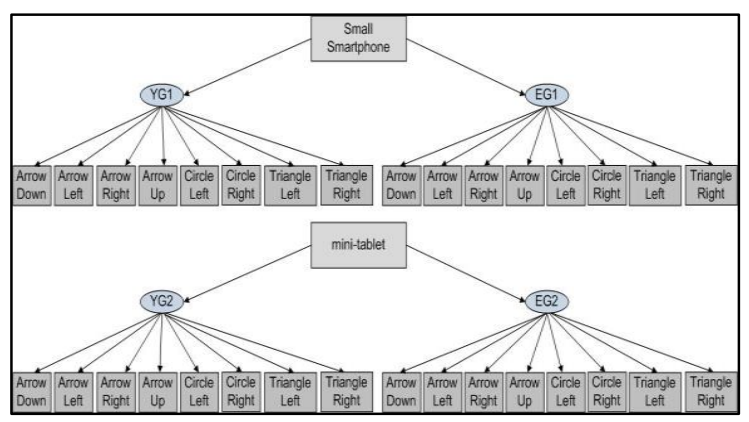

Figure 1. Organization of smartphone experiments, age groups, and 8 gestures

\section{b) Smartphones and Screen Sizes}

The two sizes of smartphones used in our research are: 1) typical small smartphones screen sizes which are between 3 and 5 inches, the smartphone used for small screen size is Samsung Galaxy Ace S 5830 - dimensions 112.4 × $59.9 \times 11.5 \mathrm{~mm}$, screen resolution: 320 x 480 pixels, screen size 3.5 inches. 2) Medium size mini-tablets screens are typically 7 inches, the smartphone used for medium screen size is Samsung Galaxy Tab 2, dimensions $193.7 \times 122.4 \times 10.5 \mathrm{~mm}$, screen resolution $1024 \mathrm{x}$ 600 with screen size 7 inches.

\section{c) Gesture Applications}

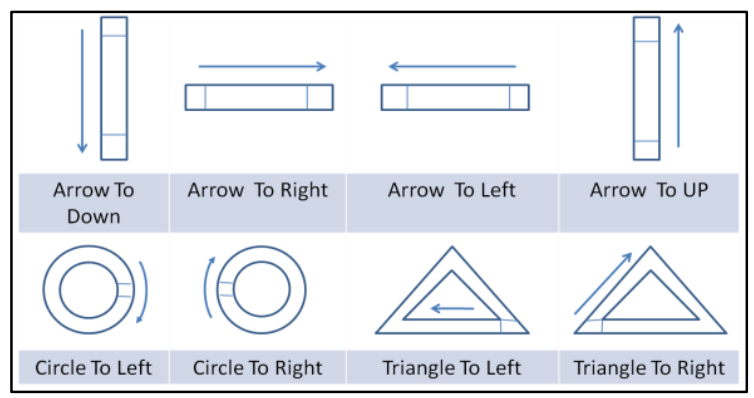


Figure 2. Gesture Applications of eight shapes

Figure 2 illustrates the eight single-line gesture shapes. These gestures were identified by Microsoft (2009), and they are similar to the single-line gestures that were used by Akl (2010), and Stößel (2009).

The single-line gestures were chosen for the following reasons: 1) Single-finger gestures were preferred by older users; 2 ) it was stated by Stößel (2012) that multi-touch based interaction in a mobile environment is not necessarily used and perceived by younger users as most natural and intuitive; 3) it was recommended by Stobel (2012) to avoid designing multi-finger gestures; 4) longer length gestures offer enough evidence of possible results when calculating gesture accuracy for having many points of the coordination $(\mathrm{x}, \mathrm{y})$ along the trajectory to match the reference data with the data obtained. This will not be available in other kinds of gestures such as tap, and pinch gesture that have short length of gesture. Longer length gesture will provide enough information about finger movement behaviour that includes accurate performing gestures, force pressure, movement time, etc.

\section{d) Participants}

The participants were selected from different age groups and include university students, university staff, and people from the local community. Details of the 50 participants took part in the experiments are described in Table 1. Each participant was asked to fill a demographic data form regarding age group, and their average experience in using smartphones. The participants' experiences of smartphone use for calling and texting were averaged based on two age groups and two screen sizes and shown in Table 1.

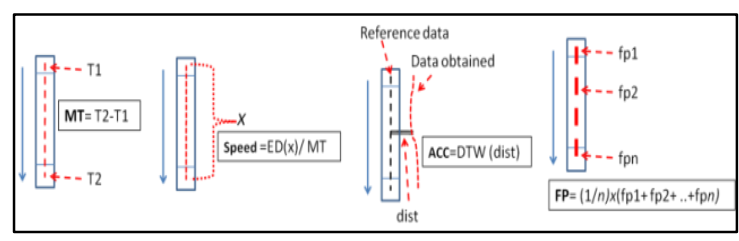

Figure 3. Illustration of how gesture metrics are calculated for the Arrow to Down gesture.

Table 1. Participants Details

\begin{tabular}{|c|c|c|c|c|c|}
\hline 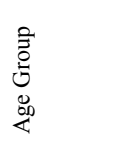 & 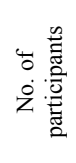 & 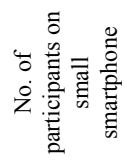 & 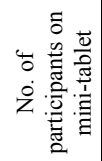 & 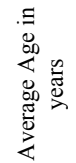 & 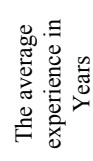 \\
\hline EG & 25 & 13 & 12 & 64.77 & 0.67 \\
\hline YG & 25 & 13 & 12 & 26.19 & 1.05 \\
\hline $\begin{array}{l}\text { Small } \\
\text { smartphone }\end{array}$ & 26 & - & - & - & 0.77 \\
\hline Mini-tablet & 24 & - & - & - & 0.95 \\
\hline
\end{tabular}

\subsection{Experimental Design and Procedure}

\section{a. Experimental Design}

The user age-group classification included 50 participants (25 Elderly and 25 younger) and the eight gestures. The participants performed each gesture six times (six trials). Therefore, the total number of gesture samples acquired was 2400 which reached to 9600 trials when we extracted the four features from each gesture sample. The four features were extracted then entered into set of processes to be used in the users' age-groups classification research.

\section{b. Experimental Procedure}

In order to conduct an experiment, each participant used a table and chair with a comfortable distance and height that suits them. A description of the experiment was given to each participant; each participant was asked to practice one gesture (i.e. rectangle to left) for 2 times to familiarize themselves with the gesture tasks. The smartphone/tablet device was placed on a table in landscape orientation to ensure consistent experiment conditions for all the participants. The participant used one hand to hold the device on the table whilst using the other hand to perform gesture tasks. This procedure was used to avoid any shaking of the smartphone/tablet that might occur if the users held the device in hand (physical ability among users vary). For example, FP could be influenced if the user pushes the screen from 
two opposite sides; downward pressure from the finger that performs gestures on the screen and upward pressure from the hand that holds the device. Note that Nicolau and Jorge (2012) used the landscape orientation, but they let their participants hold the device in their hand. In all gestures, an arrow was used as a guide to indicate the direction of the gesture. Following the instruction of Teather et al., (2010), the participants were asked to trace the complete gestures as quickly and accurately as possible.

\subsection{Gesture Features}

Four gesture metrics were used as features in this experiment: 1) Movement Time (MT, seconds), 2) Finger/Force Pressure (FP), 3) Gesture Speed (GS), and 4) Gesture Accuracy (Acc). We extracted these features by calculating each of these features for each of the six trials of each participant as follows: 1) MT is the time difference between the touch-up and touch-down; 2) Speed is the gesture length divided by MT. Euclidean distance was used to calculate the gesture length from the first point $(\mathrm{x} 1, \mathrm{y} 1)$ to the last point $(\mathrm{x} 2, \mathrm{y} 2) ; 3)$ Acc is the distance between the reference data and obtained data measured using DTW algorithm; and 4) FP is the average finger pressure for each trial calculated from finger pressure obtained at each point along the gesture. The calculations of the features are illustrated in Figure 3.

\subsection{User Age-Group Classification Process}

The classification process includes a training stage and a testing stage. The training stage is used to represent an age-group (on all screens or for a specific screen size) by preparing feature vectors for individual metrics, and depending on the experiment, a combined feature vector. The first of the six samples (trials) of a gesture was used for training.

During testing, a feature vector representing a user will be compared with the two training feature vectors (one per age group) using Euclidean distance. The user's age-group will be classified based on the nearest neighbour
(NN), since there are only two exemplars in the training dataset. Testing was performed by using the five samples of the gesture data that were not part of the training data.

The training data (i.e., the feature vectors representing the younger age-group and the elderly age-group) are calculated as follows. Calculate the average metric $\mathrm{M}_{\mathrm{i}}$ (e.g. gesture speed) of an age group (e.g. younger age-group) for a specific gesture $\mathrm{G}_{\mathrm{j}}$ (e.g. Circle-left) on a specific screen-size (e.g. small) by using the first sample of that gesture performed by each participant from that age-group. Likewise, calculate the average metric $\mathrm{M}_{\mathrm{i}}$ for the seven remaining gestures for the same screen-size and age group. This gives the feature vector for metric $M_{i}$ which consists of eight coefficients that represents an age group in the training data set. Figure 4 shows an example of how we extracted the training dataset and the testing dataset for one age group (e.g. elderly users) on the small smartphone using only one metric (e.g. MT). The feature vectors for the other three metrics are calculated the same way. We will look at the classification accuracy of individual metrics as well as combinations of them concatenate feature vectors of individual metrics to produce a combined (i.e., fused) feature vector of 32 coefficients.

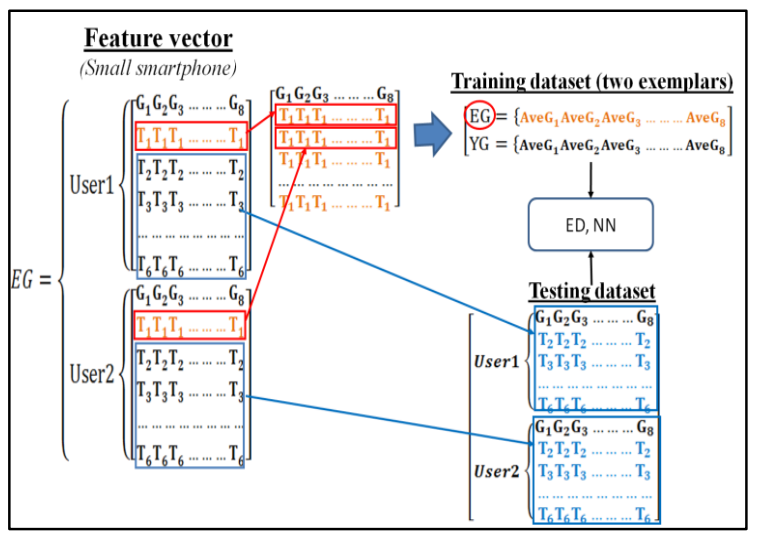

Figure 4. User age-group classification process.

We found some similar work in Hurst et al. (2008) to our research on user age-group classification research. However, our research is 
different to Hurst et al. in three main areas. First, they based their measurement on PC mouse movements, whereas we used finger-based touch-gestures on smartphones. Second, their study used the following metrics: 1) the movement time needed to complete the task, and 2) the number attempts needed to perform a task correctly. Whereas the metrics used in our research are: gesture accuracy, gesture speed, FP, and MT. Also, we used dynamic time warping (DTW), and Euclidean distance (ED) to calculate gesture accuracy, and gesture lengths respectively to calculate speed. Finally, Hurst et al. used decision trees based on the observation in the statistical analysis, whereas in our research, we used nearest-neighbour $(\mathrm{NN})$ with ED to classify user's age-group.

\section{RESULTS AND DISCUSSIONS}

Three scenarios were considered to evaluate the possibility of classifying user age based on touch-gesture features: user-dependent $(100 \%$, i.e. all participants included in training data); user-independent with $50 \%$ training data; and user-independent with data from only one participant per age-group used as training data. The explanation given in the previous section was for training data relevant to user-dependent scenario, i.e., all participants from each age group were used in the training datasets. We used only half of the participants from each age group in the training datasets for userindependent with $50 \%$ training data as shown in Figure 5Error! Reference source not found.. Likewise, we used only one participant from each age group in the training datasets for userindependent with data from one participant as training data. We evaluated the classification accuracy results of individual metrics as well as their combinations to measure the influence of gesture features on the age-group classification accuracy on different screen sizes and under difference scenarios.

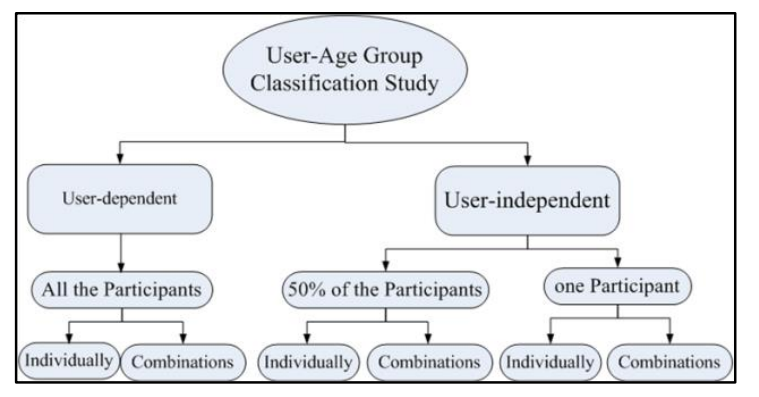

Figure 5. Organization of Users age-groups classification research

\subsection{User-Dependent Age-group Classification (training with all participants)}

\section{a. Effect of Age}

With a single feature, the highest classification accuracy (94\%, see arrow-up) was achieved with MT for younger users, whilst gesture accuracy (Acc) resulted in the lowest classification accuracy of $39 \%$ for elderly users (see arrow-down, in Table 2).

With combined features, the highest classification accuracy of $94 \%$ was achieved with Acc, FP, and MT for younger users. The lowest classification accuracy (52\%) was achieved with Acc, FP, and MT for elderly users. In general, using a combination of gesture-based features has had a positive effect on the classifications accuracy for YG - the classification accuracy improved from $74 \%$ to $86 \%$. Also, the lowest classification accuracy for EG improved from $39 \%$ to $52 \%$.

\section{b. Screen Size Influence}

With a single feature, GS achieved the highest classification accuracy of $85 \%$ (see arrow-up) for the users of the two age groups on small smartphone, whilst the Acc resulted in the lowest classification accuracy (53\%, see arrowdown) on the mini-tablet (see Table 2)Error! Reference source not found..

With combined features, the highest classification accuracy of $86 \%$ was achieved on the small smartphone with Acc, FP, and GS. The lowest classification accuracy (58\%) was achieved on the mini-tablet with Acc, FP, and GS. 


\subsection{User-Independent Classification (training with $50 \%$ of participants)}

In this experiment, we wanted to evaluate the age-group classification accuracy based on participants that were not in the training set to simulate real-life application scenarios. For example, a user interacting with a smartphone/tablet device at a shopping centre to find product information - users gesture samples are unavailable in advance to train the system. In this experiment scenario, training data consist of only $50 \%$ of the participants from each age group. The results presented in Table 3 show that the system can classify the user's age-group with no significant difference in classification accuracy between user-independent and userdependent scenarios.

\section{a. Effect of Age}

With a single feature, the highest classification accuracy (90\%, see arrow-up) was achieved with Acc for younger users, whilst GS achieved the highest classification accuracy of $61 \%$ (see arrow-up) for elderly users (see Table 3)Error! Reference source not found..

With combined features, the highest classification accuracy (57\%) for elderly users was achieved with FP, MT, and GS. the highest classification accuracy of $91 \%$ was achieved with two different of combinations for younger users: 1) Acc, and FP, 2) Acc, FP, and GS. The lowest classification accuracy (46\%) was achieved with Acc, and GS for elderly users.

\section{b. Screen Size Influence}

When using a single feature, GS achieved the highest classification accuracy of $85 \%$ (see arrow-up) on the small smartphone, whilst Acc resulted in the lowest classification accuracy (53\%, see arrow-down) on the mini-tablet (see Table 3).
With combined features, the highest classification accuracy (82\%), on the small smartphone was achieved with Acc and GS. The lowest, on mini-tablet, classification accuracy (53\%) was based on Acc, and GS.

\subsection{User Independent (training with participant)}

In this experiment, we wanted to evaluate the scenario where only a limited number of participants are available to collect training data. Therefore, we used the gesture data of one participant from each age group to create the training datasets. The results presented in Table 4Error! Reference source not found. shows that the system can classify users' agegroup using a small number of training data and still achieve a reasonable classification accuracy compared to user-dependent $(100 \%)$ or userindependent $(50 \%)$ scenarios we presented earlier.

\section{a.Effect of Age}

In analysing single features, the highest classification accuracy (96\%, see arrow-up) was achieved with MT for younger users, whilst the MT resulted in the lowest classification accuracy of $42 \%$ (see arrow-down) for elderly users.

\section{Error! Reference source not found.}

With combined features, the highest classification accuracy of $97 \%$ was achieved with Acc, FP, and MT for younger users. The lowest classification accuracy (42\%) was achieved with Acc, FP and MT for elderly users.

\section{b. Screen Size Influence}

In analysing single features, GS achieved the highest classification accuracy of $86 \%$ (see arrow-up) on the small smartphone, whilst Acc resulted in the lowest classification 
accuracy (56\%, see arrow-down) on mini-tablet (see Table 4).

With combined features, the highest smartphone was achieved with Acc, FP, and GS. The lowest classification accuracy (58\%) was based on Acc and FP on mini-tablet.

\begin{tabular}{|l|l|l|l|l|l|}
\hline \multirow{1}{*}{ Analysis } & \multicolumn{1}{|c|}{ Feature } & \multicolumn{2}{c|}{ Screen Sizes } & \multicolumn{2}{c|}{ Age Groups } \\
\cline { 3 - 6 } & & $\begin{array}{c}\text { Small } \\
\text { Smartphone }\end{array}$ & Mini-tablet & \multicolumn{1}{|c|}{ YG } & \\
\hline \multirow{5}{*}{ Individually } & Speed & $85.38 \% \uparrow$ & $59.17 \%$ & $63.20 \% \uparrow$ & $82.40 \%$ \\
\cline { 2 - 6 } & Gesture accuracy & $78.46 \%$ & $52.50 \% \downarrow$ & $39.20 \% \downarrow$ & $92.80 \%$ \\
\cline { 2 - 6 } & FP & $62.31 \% \downarrow$ & $70.00 \% \uparrow$ & $58.40 \%$ & $73.60 \% \downarrow$ \\
\cline { 2 - 6 } & MT & $75.38 \%$ & $69.17 \%$ & $50.40 \%$ & $94.40 \% \uparrow$ \\
\hline \multirow{5}{*}{ Combinations } & Gesture accuracy, FP & $80.77 \%$ & $63.33 \%$ & $52.00 \%$ & $92.80 \%$ \\
\cline { 2 - 6 } & Gesture accuracy, FP, Speed & $86.15 \% \uparrow$ & $57.50 \% \downarrow$ & $59.20 \% \uparrow$ & $85.60 \% \downarrow$ \\
\cline { 2 - 6 } & Gesture accuracy, FP, MT & $76.92 \% \downarrow$ & $69.17 \% \uparrow$ & $52.00 \% \downarrow$ & $94.40 \% \uparrow$ \\
\cline { 2 - 6 } & Gesture accuracy, FP, MT, Speed & $80.00 \%$ & $66.67 \%$ & $55.20 \%$ & $92.00 \%$ \\
\cline { 2 - 6 } & Gesture accuracy, Speed & $83.85 \%$ & $58.33 \%$ & $56.80 \%$ & $86.40 \%$ \\
\cline { 2 - 6 } & FP, MT, Speed & $80.00 \%$ & $63.33 \%$ & $56.00 \%$ & $88.00 \%$ \\
\cline { 2 - 6 } & MT, Speed & $80.00 \%$ & $65.00 \%$ & $56.00 \%$ & $89.60 \%$ \\
\hline
\end{tabular}

classification accuracy $(90 \%)$, on the small

Table 2. User's age-group classifications results for user-dependent (100\%) of ageing influence. Arrow up shows larger value and arrow down shows lower values

\begin{tabular}{|c|c|c|c|c|c|}
\hline \multirow[t]{2}{*}{ Analysis } & \multirow[t]{2}{*}{ Feature } & \multicolumn{2}{|c|}{ Screen Sizes } & \multicolumn{2}{|c|}{ Age Groups } \\
\hline & & $\begin{array}{c}\text { Small } \\
\text { smartphone }\end{array}$ & Mini-tablet & $E G$ & $Y G$ \\
\hline \multirow[t]{4}{*}{ Individually } & Speed & $84.62 \% \uparrow$ & $57.50 \%$ & $60.80 \% \uparrow$ & $82.40 \%$ \\
\hline & Gesture accuracy & $81.54 \%$ & $53.33 \% \downarrow$ & $45.60 \% \downarrow$ & $90.40 \% \uparrow$ \\
\hline & FP & $61.54 \% \downarrow$ & $70.83 \% \uparrow$ & $56.80 \%$ & $75.20 \% \downarrow$ \\
\hline & MT & $79.23 \%$ & $65.00 \%$ & $55.20 \%$ & $89.60 \%$ \\
\hline \multirow[t]{3}{*}{ Combinations } & Gesture accuracy, and FP & $81.54 \%$ & $61.67 \%$ & $52.80 \%$ & $91.20 \% \uparrow$ \\
\hline & Gesture accuracy, FP, Speed & $81.54 \%$ & $62.50 \%$ & $53.60 \%$ & $91.20 \% \uparrow$ \\
\hline & Gesture accuracy, FP, MT & $81.54 \%$ & $62.50 \%$ & $56.00 \%$ & $88.80 \%$ \\
\hline
\end{tabular}

Table 3. Age-group classifications results for User-Independent metrics (50\%) of ageing influence. Arrow up shows larger value and arrow down shows lower values

Table 4. User's age-group classifications results for User-Independent metrics (1 participant) of ageing influence

\begin{tabular}{|l|l|l|l|l|l|}
\hline \multirow{1}{*}{ Analysis } & \multicolumn{1}{c|}{ Features (metrics) } & \multicolumn{1}{c|}{ Screen Sizes } & \multicolumn{2}{c|}{ Age Groups } \\
\cline { 3 - 6 } & & $\begin{array}{c}\text { Small } \\
\text { smartphone }\end{array}$ & mini-tablet & \multicolumn{1}{|c|}{ YG } & \\
\hline \multirow{5}{*}{ Individually } & Speed & $86.15 \% \uparrow$ & $59.17 \%$ & $74.40 \% \uparrow$ & $72.00 \% \downarrow$ \\
\cline { 2 - 6 } & Gesture accuracy & $66.15 \%$ & $55.83 \% \downarrow$ & $45.60 \%$ & $76.80 \%$ \\
\cline { 2 - 6 } & FP & $65.38 \% \downarrow$ & $70.00 \% \uparrow$ & $52.00 \%$ & $83.20 \%$ \\
\cline { 2 - 6 } & MT & $77.69 \%$ & $60.00 \%$ & $42.40 \% \downarrow$ & $96.00 \% \uparrow$ \\
\hline Combinations & Gesture accuracy, and FP & $76.92 \% \downarrow$ & $57.50 \% \downarrow$ & $43.20 \%$ & $92.00 \%$ \\
\cline { 2 - 6 } & Gesture accuracy, FP, Speed & $90.00 \% \uparrow$ & $59.17 \%$ & $69.60 \% \uparrow$ & $80.80 \%$ \\
\hline
\end{tabular}




\begin{tabular}{|l|l|l|l|l|l|}
\hline \multirow{4}{*}{} & Gesture accuracy, FP, MT & $77.69 \%$ & $60.83 \% \uparrow$ & $42.40 \% \downarrow$ & $96.80 \% \uparrow$ \\
\cline { 2 - 6 } & Gesture accuracy, FP, MT, Speed & $88.46 \%$ & $60.83 \% \uparrow$ & $56.80 \%$ & $93.60 \%$ \\
\cline { 2 - 6 } & Gesture accuracy, Speed & $85.38 \%$ & $58.33 \%$ & $69.60 \% \uparrow$ & $75.20 \% \downarrow$ \\
\cline { 2 - 6 } & FP, MT, Speed & $87.69 \%$ & $60.83 \% \uparrow$ & $56.00 \%$ & $93.60 \%$ \\
\cline { 2 - 5 } & MT, Speed & $85.38 \%$ & $60.83 \% \uparrow$ & $53.60 \%$ & $93.60 \%$ \\
\hline
\end{tabular}

found to be relatively similar for the different age groups. In general, taking a combination of gesture-based features has had a positive effect

In general, the above results from the three different scenarios reveal that the classification accuracy is higher for younger users than it is for elderly users- a significant number of elderly users were misclassified as belonging to the younger age-group. This is because the gesture performance of most younger users were similar to each other, where as some elderly users, most likely due to their experience in using smartphones, were able to perform gestures with similar characteristics to younger users. This indicates that the performance of some elderly users was similar to the younger users' performance. Note that Minocha et al. (2013) concluded that not all elderly people are vulnerable in performing technology tasks.

Our research provides evidence for the possibility of classifying user's age-group based on touch-gestures. Also, combining gesturebased features had a positive effect on the classification accuracy. For example, in the last scenario (i.e. one participant in the training dataset) for younger users, the highest classification accuracy with a single feature increased from $96 \%$ to $97 \%$. In the last scenario (i.e. one participant in the training dataset), the lowest classification for younger users the accuracy improved from $72 \%$ to $75 \%$.

The results demonstrated that the classification accuracy is higher on the small smartphone than it is on the mini-tablet. This is because, as we found when extracting the four metrics, the gesture performance on the small smartphone was different for the two age-groups - elderly were particularly slow, less accurate and exerted more pressure on the screen than the younger users (REF to the thesis). Moreover, the gesture performance on large screen sizes was on the classification accuracy. For example in the last scenario (i.e. one participant in the training dataset) - on the small smartphone, the highest classification accuracy with a single feature increased from $86 \%$ to $90 \%$ and the lowest classification accuracy with a single feature improved from $65 \%$ to $77 \%$. In last scenario (i.e. one participant in the training dataset), the lowest classification accuracy on mini-tablets improved from $56 \%$ to $58 \%$.

The research provided evidence for the possibility of classifying users' age-group based on touch-gestures, even if the numbers of participants in training datasets were very small, as the results presented in Table 4 Error! Reference source not found.showed that the system can still achieve a reasonable classification accuracy compared to userdependent $(100 \%)$ or user-independent $(50 \%)$ scenarios we presented earlier.

\section{CONCLUSION}

This paper investigated the possibility of classifying users' age-group using touch-gesture based features on smartphones. We proposed the use of touch-gesture based features on smartphones to classify users' age-group. Four gesture-based metrics (i.e., gesture accuracy, gesture speed, movement time, and finger pressure), were used as discriminant features represent age-groups. Nearest Neighbour classification was used to classify a given user's age-group.

We analysed the accuracy of the four metrics individually as well as in combination to evaluate their ability to distinguish users as belonging to one of the two age-groups. Our analysis showed that age-group classification based on the four features is higher on the small 
smartphone compared the mini-tablet size. On the other hand, less significant differences in gesture performance were found for the two age groups on the mini-tablet. The age-classification results show that it was relatively easier to classify younger users than the older ones. Using a combination of gesture features in the classification process improved the classification accuracy when compared to using a single feature.

Classification accuracy for userdependent and user-independent scenarios were considered. The results for all three scenarios remained close to each other indicating that the age-group classification can be performed with reasonable accuracy by using only a small number of training samples and these do not have to belong to the user. The results in the three scenarios provided evidence for the possibility of classifying users' age based on touch-gestures. To the best of our knowledge we have not come across any study conducted on smartphones to classify age-group using gesturebased features.

A comprehensive set of experiments with detailed analysis is required to identify other useful gesture based features for agegroup classification and understand the effect of user experience on age classification accuracy. This forms the future direction of our research.

\section{REFERENCES}

AL-SHOWARAH SA. Effects of Age on Smartphone and Tablet Usability, based on Eyemovement Tracking and Touch-gesture Interactions. The University of Buckingham, $\mathrm{PhD}$ thesis 2015: School of Science and Postgraduate Medicine, Buckingham - UK. 2015.

ARNOTT J, KHAIRULLA Z, DICKINSON A, SYME A, ALM N, EISMA R \& GREGOR P. E-mail interfaces for older people. Dundee, Scotland, U.K, IEEE International Conference on Systems, Man and Cybernetics. University of Dundee. 2004; 1: 111 -117.

AKL A \& VALAEE S. Accelerometer-based gesture recognition via dynamic-time warping, affinity propagation, \& compressive sensing. IEEE International Conference on Acoustics Speech and Signal Processing (ICASSP). 2010; 2270-2273.

HURST A, HUDSON SE, MANKOFF J \& TREWIN S. Automatically detecting pointing performance. Proceedings of the 13th international conference on Intelligent user interfaces. ACM. 2008; 11-19.

KEATES S \& TREWIN S. Effect of age and Parkinson's disease on cursor positioning using a mouse. s.l., s.n. 2005; 68-75.

MICROSOFT. Application gestures and semantic behavior. 2009. Available from: https://msdn.microsoft.com/enus/library/windo ws/desktop/ms704830(v=vs.85).aspx (Accessed on 27 January 2015).

MINOCHA S, HARTNETT E, DUNN K, EVANS ST, MIDDUP C, MURPHY B \& ROBERTS D. Conducting empirical research with older people. In: Designing for- and with vulnerable people, 27 April 2013, Paris, France. 2013.

MOFFATT K \& MCGRENERE J. Steadiedbubbles: Combining techniques to address penbased pointing errors for younger and older adults. Proceedings of the SIGCHI Conference on Human Factors in Computing Systems. ACM. 2010; 1125-1134.

NICOLAU H \& JORGE J. Elderly text-entry performance on touchscreens. Proceedings of the 14th international ACM SIGACCESS conference on Computers and accessibility. ACM. 2012;127-134. 
PATTISON M \& STEDMON AW. Inclusive Design and Human Factors: Designing Mobile Phones for Older Users. PsychNology Journal. 2006; 4(3): 267-284.

STÖßEL C, WANDKE H \& BLESSING L. An evaluation of finger-gesture interaction on mobile devices for elderly users. Prospektive Gestaltung von Mensch-Technik-Interaktion. 2009; 8: 470-475.

STÖßEL C. Gestural Interfaces for Elderly User - Help or Hindrance? Faculty of Sciences, Mechanical Engineering and Transport Systems, The Technical University of Berlin, $\mathrm{PhD}$ thesis 2012: The Technical University of Berlin. Faculty of Sciences, Mechanical Engineering and Transport Systems. PhD thesis 2012.

SULTANA A \& MOFFATT K. Automatic Error Detection from Pointing Device Input Data. 2013. Available from: https://www.asis.org/asist2013/proceedings/sub missions/posters/46poster.pdf (Accessed on 27 January 2015).

TEATHER RJ, NATAPOV D \& JENKIN M. Evaluating haptic feedback in virtual environments using ISO 9241--9. Virtual Reality Conference (VR). IEEE. 2010; 307-308. 